\title{
Topological and trivial magnetic oscillations in nodal loop semimetals
}

\author{
László Oroszlány, ${ }^{1}$ Balázs Dóra, ${ }^{2}$ József Cserti, ${ }^{1}$ and Alberto Cortijo ${ }^{3}$ \\ ${ }^{1}$ Department of Physics of Complex Systems, Eötvös University, H-1117 Budapest, Hungary \\ ${ }^{2}$ Department of Theoretical Physics and MTA-BME Lendület Spintronics Research Group (PROSPIN), \\ Budapest University of Technology and Economics, 1521 Budapest, Hungary \\ ${ }^{3}$ Materials Science Factory, Instituto de Ciencia de Materiales de Madrid, CSIC, Cantoblanco, 28049 Madrid, Spain
}

(Received 19 January 2018; published 8 May 2018)

\begin{abstract}
Nodal loop semimetals are close descendants of Weyl semimetals and possess a topologically dressed band structure. We argue by combining the conventional theory of magnetic oscillation with topological arguments that nodal loop semimetals host coexisting topological and trivial magnetic oscillations. These originate from mapping the topological properties of the extremal Fermi surface cross sections onto the physics of two dimensional semi-Dirac systems, stemming from merging two massless Dirac cones. By tuning the chemical potential and the direction of magnetic field, a sharp transition is identified from purely trivial oscillations, arising from the Landau levels of a normal two dimensional (2D) electron gas, to a phase where oscillations of topological and trivial origin coexist, originating from 2D massless Dirac and semi-Dirac points, respectively. These could in principle be directly identified in current experiments.
\end{abstract}

DOI: 10.1103/PhysRevB.97.205107

\section{INTRODUCTION}

Topological nodal semimetals are three dimensional semimetallic systems where the valence and conduction bands closest to the Fermi level cross each other in momentum space. In the case of Weyl/Dirac semimetals, the crossing consists of a discrete set of points, while in the case of nodal loop semimetals (NLSM), the crossing takes the form of a closed loop [1]. While both families of semimetals are topologically nontrivial, the nature of the topological structure is quite different. In the case of a NLSM, the 1D character of the line of singularities (that ultimately comes from the discrete symmetries of the system [2]) determines the topological invariant similar to 1D topological insulators [3]: any closed path in momentum space along which the Hamiltonian of the system is gapped can be threaded by the loop an odd or even (including zero) number of times. In the former case, this closed path acquires a Berry phase of $\pi$, while in the latter this Berry phase is zero, defining in this way a $Z_{2}$ invariant $[2,4]$. A fundamentally intriguing question is whether it is possible to observe this $Z_{2}$ topological character in experimentally accessible properties in NLSMs. In the case of Weyl semimetals there is a nontrivial Berry curvature (not appearing in NLSM) that directly modifies transport and optical properties [5]. For the case of NLSMs, it has been suggested that this topological structure should manifest in quantum oscillations $[4,6,7]$ or through the presence of surface states $[8,9]$.

The recent theoretical effort to identify NLSM candidates [10-16] was accompanied by intense experimental progress, mostly focused on ARPES [17-22] yielding surface properties and magnetotransport experiments [23-28] sensitive to bulk characteristics, particularly in the family of $\mathrm{ZrSi}$ chalchogenides. Although recent experiments show the presence of a nontrivial Berry phase [25-28], to this day it is unclear how the crossover between trivial and topological oscillations is manifested in magneto oscillation spectra [29]. It is thus crucial to develop a theoretical approach to expose the fingerprints of the topological nature of these novel materials.

In this work we present a comprehensive theoretical description of magnetic oscillations present in these novel systems. Based on simple topological arguments backed by a semiclassical analysis we construct the phase diagram for finite chemical potential and arbitrary field orientation and contrast it to numerical calculations.

\section{TOPOLOGICAL CONTENT OF MAGNETIC OSCILLATIONS}

The effects of an external magnetic field can be qualitatively understood by recalling that the electronic motion is confined in the plane perpendicular to the magnetic field. Upon fixing the chemical potential of the electron, it follows the trajectory set by the cross section of the constant energy contour and the plane perpendicular to the magnetic field. By sweeping the magnetic field, the extremal orbits among these trajectories determine the characteristic frequency of magnetic oscillations [30,31], simply because their contribution dominates over the other orbits. This gives rise to the celebrated de Haas-van Alphen effect and the Shubnikov-de Haas oscillations, which are well documented for normal metals, with both quantities being intimately related to the evolution of the density of states (DOS) when the magnetic field is varied. The quantization of the cyclotron orbits is expressed in terms of the Onsager quantization condition

$$
\frac{\mathcal{A}[E] \hbar}{e B}=2 \pi(n+\gamma),
$$

where $\mathcal{A}[E]$ is the area enclosed by the cyclotron orbit in momentum space for energy $E, n$ is an integer, $B$ is the applied magnetic field, and $\pi(1-2 \gamma)$ is the Berry phase accumulated 
by the cyclotron orbit [32,33]. Equation (1) implicitly defines the Landau level spectrum [30]. As mentioned in the Introduction, the presence of nontrivial Berry phases are expected for extremal orbits threading the nodal loop. Thus the $Z_{2}$ invariant assigned to the cyclotron orbits can be identified with $\gamma$ [17]. The presence of cyclotron orbits with nontrivial Berry phases has also been linked to the appearance of an almost-flat zero energy Landau level [6], similar to the case of graphene [34,35].

\section{BAND STRUCTURE OF NODAL LOOP SEMIMETALS}

We consider the low energy Hamiltonian of a NLSM as

$$
H=\left(\Delta-\frac{p_{\perp}^{2}}{2 m}\right) \sigma_{x}+v p_{z} \sigma_{z}=\mathbf{d}(\mathbf{p}) \cdot \boldsymbol{\sigma},
$$

where the $\sigma$ 's are Pauli matrices, $p_{\perp}^{2}=p_{x}^{2}+p_{y}^{2}, m>0$ is an effective mass, $\Delta$ is an energy scale, and $v$ is the Fermi velocity in the $z$ direction. Note that the orientation pattern of $\mathbf{d}(\mathbf{p})$ encodes the topological properties of the system. The Hamiltonian can readily be diagonalized to yield the spectrum as $E_{ \pm}(\mathbf{p})= \pm \sqrt{v^{2} p_{z}^{2}+\left(\Delta-\frac{p_{\perp}^{2}}{2 m}\right)^{2}}$. The absence of $\sigma_{y}$ signifies the chiral symmetry of the considered model. The presence of this symmetry is required to stabilize the nodal loop in Eq. (2). This also guarantees the quantized Berry phases. For $\Delta>0$, the Fermi surface consists of a circle in the $x-y$ plane with radius $\sqrt{2 \Delta m}$. In the presence of finite chemical potential, $\mu>0$, the Fermi surface is determined from the $\mu=\epsilon_{+}(\mathbf{p})$ relation, and the Fermi surface evolves from a circle at $\mu=0$ to a toruslike surface for finite $\mu$, whose tube radius is set by the chemical potential. For the present model in Eq. (2), two qualitatively different characteristic cases arise for the quantum oscillations by considering a magnetic field parallel and perpendicular to the $z$ axis.

\section{EXTREMAL FERMI SURFACE CROSS SECTIONS}

First we discuss the magnetic field in the $z$ direction, thus restricting electrons to the $x-y$ plane. The extremal cross section of the Fermi surface is depicted in Fig. 1(a). In this case the effective Hamiltonian, Eq. (2), is dominated by its first term and $\mathbf{d}(\mathbf{p})$ points always in the $x$ direction; hence the spinor structure is prevented from acquiring any winding. The emerging magnetic oscillations are thus doomed to be trivial with Berry phase zero and $\gamma=1 / 2$.

Turning the magnetic field perpendicular to the $z$ axis, the effective motion of electrons is confined into vertical cuts through the torus: depending on the position of the cutting plane, cross sections can either be a single loop or two disconnected closed rings. The resulting physics is dictated by a semi-Dirac point in 2D [36,37] with an effective Hamiltonian in the $y-z$ plane as

$$
H_{\mathrm{eff}}=\left(\tilde{\Delta}-\frac{p_{y}^{2}}{2 m}\right) \sigma_{x}+v p_{z} \sigma_{z},
$$

where $\tilde{\Delta}=\Delta-\frac{p_{x}^{2}}{2 m}$ and the magnetic field points in the $x$ direction. For $\tilde{\Delta}=0$, a semi-Dirac point is realized with a combination of quadratic and linear dispersions (in the $y$ and $z$ directions), respectively. When $\tilde{\Delta}<0$, the spectrum is
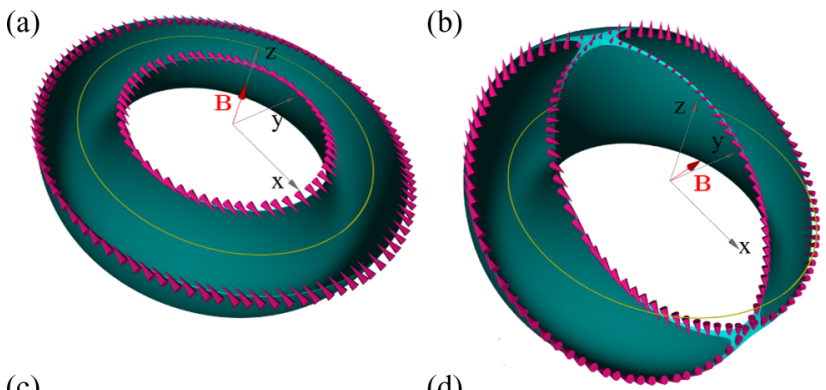

(c)
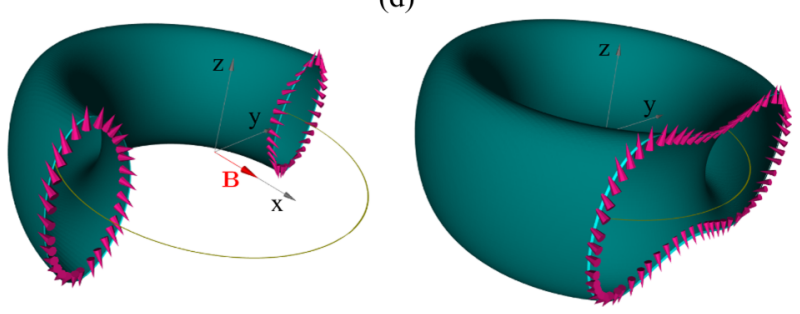

FIG. 1. Evolution of the cross sections by planes perpendicular to magnetic field of the torus Fermi surface is shown: (a) magnetic field in $z$ direction; the winding of the vector $\mathbf{d}(\mathbf{p})$ (magenta cones) reveals topologically trivial character. Panel (b) shows the case of critical angle, when the Lifshitz transition occurs. The (c) and (d) panels depict the topological and trivial extremal cross sections for magnetic field perpendicular to $z$ axis, the winding of the vector $\mathbf{d}(\mathbf{p})$ signal $\pm \pi$, and zero Berry phases, respectively.

gapped; the dispersion above the gap is reminiscent to that of a $2 \mathrm{D}$ anisotropic mormal electron gas, thus topologically trivial. Finally, the most important situation from a topological point of view arises for $\tilde{\Delta}>0$, when the cross section by a plane perpendicular to the magnetic field hosts two linearly dispersing Dirac cones, carrying a Berry phase of $\pm \pi$.

For a given chemical potential, the extremal cross sections occur in two different locations of the cutting plane: there is a single, connected, topologically trivial, maximal Fermi surface and there are two, disconnected, topological (due to the $\pm \pi$ Berry phases), minimal Fermi surfaces, visualized in Figs. 1(d) and 1(c), respectively. These Fermi surface sections determine the magnetic oscillations, which will be a mixture of topological and nontopological frequencies, stemming from the aforementioned disconnected and connected extremal Fermi surfaces, respectively. Their topological content is revealed by following the winding of vector $\mathbf{d}(\mathbf{p})$ in Eq. (2) upon going around a closed cross section. In the case of the connected, maximal cross section, we can unwind $\mathbf{d}(\mathbf{p})$ to point in the same direction for all $\mathbf{p}$ around the cross section by plane, thus representing a topologically trivial surface. On the other hand, for the two disconnected, minimal area Fermi surfaces cross sections, the winding of $\mathbf{d}(\mathbf{p})$ is identical to that of graphene [38,39], going around clockwise and counterclockwise in the two disconnected cross sections, giving rise to Berry phases $\pm \pi$.

For a general magnetic field tilted by an angle $\vartheta$ from the $z$ axis, there is a sharp transition between only nontopological and a combination of topological and nontopological magnetic oscillations. For small $\vartheta$, the extremal Fermi surfaces are still topologically trivial: the extremal Fermi surfaces corresponding to $\vartheta=0$ are adiabatically connected to the case of small 

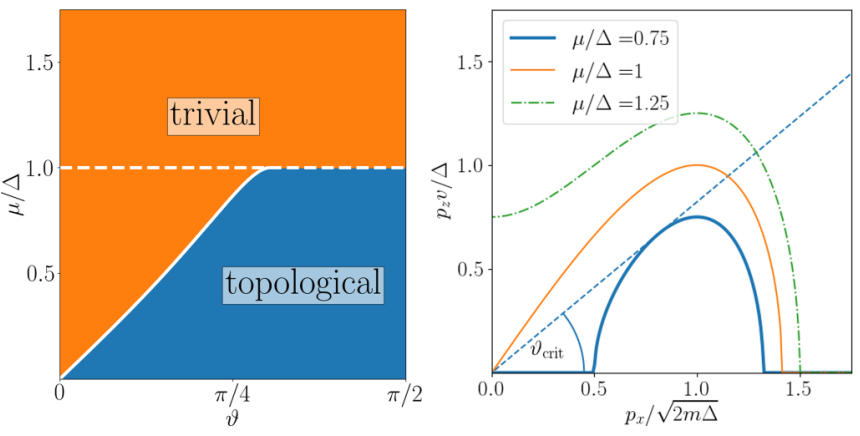

FIG. 2. Phase diagram of Eq. (2) is shown as a function of energy (chemical potential) and magnetic field angle (left panel) for $\Delta=$ $2 v^{2} m$. At a fixed small energy, the oscillations are nontopological for small tilt angles and become a mixture of topological and trivial oscillations upon tilting the magnetic field further from the $z$ axis. The right panel, visualizing a quarter of a cross section of the Fermi surface, depicts the geometric interpretation of the critical tilt angle in the $p_{y}=0$ plane, $\vartheta_{\text {crit }}$ growing with the chemical potential. When the Fermi surface becomes a horn torus at $\mu=\Delta$, only topologically trivial cross sections exist.

finite tilting angle; therefore, their topologically trivial nature remains unchanged. At a critical angle

$$
\tan \left(\vartheta_{\text {crit }}\right)=(v \sqrt{m})^{-1} \sqrt{\Delta-\sqrt{\Delta^{2}-\mu^{2}}},
$$

a Lifshitz transition occurs and, for larger angles, the extremal Fermi surfaces are qualitatively similar to the 2D semi-Dirac case. These are adiabatically connected to the $\vartheta=\pi / 2$ case and contain topological (with Berry phase $\pm \pi$ ) and nontopological (with Berry phase zero) features. Finally, for large chemical potential, another transition occurs and the ring torus shaped Fermi surfaces in Fig. 1 change into spindle torus, in which case even the previously disconnected Fermi surface loops touch and become topologically trivial. All these features are summarized in Fig. 2. Note that the quantization of the Berry phase to $\pi$ and zero is guaranteed by the same symmetry that protects the zero energy nodal line.

\section{SEMICLASSICAL ANALYSIS}

Having determined the general structure in magnetic field oscillations from the extremal Fermi surface cuts, we turn to a more quantitative analysis by inserting a finite magnetic field into Eq. (2). The orbital effects of an external magnetic field in the $x-z$ plane are captured by the vector potential $\mathbf{A}=$ $B(0, \cos (\vartheta) x-\sin (\vartheta) z, 0)$, where $\vartheta$ is measured from the $z$ axis, using the Peierls substitution, $\mathbf{p} \rightarrow \mathbf{p}-e \mathbf{A}$ in Eq. (2) [41]. For a magnetic field in the $z$ direction, $\vartheta=0$, the spectrum reads as [7] $E_{ \pm, n, p_{z}}= \pm \sqrt{\left(\Delta-\frac{e B}{m}(n+1 / 2)\right)^{2}+v^{2} p_{z}^{2}}$ and the extremal cross section occurs at $p_{z}=0$. The appearance of the $1 / 2$ signals the absence of a finite Berry's phase and the expected trivial nature for oscillations.

Consider now the opposite limiting case, namely $\vartheta=\pi / 2$, that is the magnetic field lying in the $x$ direction. Although the resulting Hamiltonian cannot be diagonalized analytically, its spectral properties can be elucidated by a semiclassical

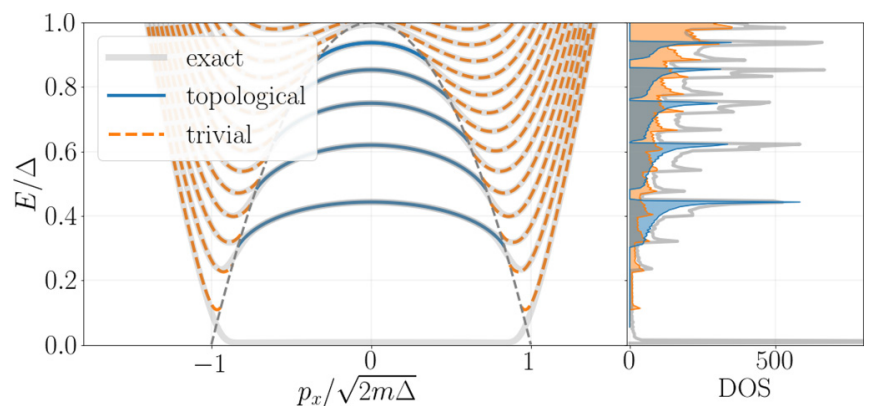

FIG. 3. Spectrum of the in plane magnetic configuration obtained by exact diagonalization and by the semiclassical approach for $\sqrt{2 m} \Delta^{3 / 2} / v e B=20$. The dashed gray lines signal the boundary between the topological and trivial part of the spectrum. The corresponding DOS from numerics as well as the separate topological and trivial contributions from semiclassics is plotted and is dominated by the topological contribution at small energies.

approach similar to Ref. [36]. This yields the spectrum, $E_{n, p_{x}}$, in the trivial and topological regimes from

$$
\begin{aligned}
\frac{3 \pi}{2 \sqrt{2}}\left(n+\frac{1}{2}\right) & =\sqrt{\epsilon}(2 \alpha E(x)+(\epsilon-\alpha) K(x)), \\
\frac{3 \pi}{2} n & =\sqrt{\alpha+\epsilon}\left(\alpha E\left(x^{-1}\right)+(\epsilon-\alpha) K\left(x^{-1}\right)\right),
\end{aligned}
$$

where $K(x)$ and $E(x)$ are the complete elliptic integrals [42] of the first and second kind, respectively, $x=(\epsilon+\alpha) / 2 \epsilon, \alpha=$ $\tilde{\Delta}\left(2 m /(v e B)^{2}\right)^{1 / 3}, \epsilon=E_{n, p_{x}}\left(2 m /(v e B)^{2}\right)^{1 / 3}$, and $n$ is a nonnegative integer integer. The upper/lower equation corresponds to the trivial/topological part of the spectrum, respectively, and their boundary is at $\alpha=\epsilon$. The extremal parts of the spectrum (in $p_{x}$ ) for the topological and trivial regions read as

$$
E_{n} \approx \begin{cases} \pm 2 \sqrt{\sqrt{\Delta} v e B n / \sqrt{2 m}}, & \text { topological }, \\ \pm\left(\frac{v e B}{\sqrt{m}}\left(n+\frac{1}{2}\right)\right)^{2 / 3}, & \text { trivial, }\end{cases}
$$

signaling a Berry phase of $\pi$ and zero, respectively. These correspond to the Landau levels of two dimensional massless Dirac [39] and semi-Dirac [37] points, respectively.

All these features, including the Berry phases, are reproduced from a numerical solution of Eq. (2) in a magnetic field, shown in Fig. 3. Apart from very small energies or chemical potential, the Onsager quantization approximates perfectly the numerically obtained spectrum.

\section{DENSITY OF STATES}

The DOS (density of states) is given by $g(E)=$ $\sum_{n, p_{y}, k} \delta\left(E-E_{n, k}\right)$ with $k$ the appropriate quantum numbers for a given magnetic field orientation. For magnetic field in the $x-y$ plane, the $p_{x}$ dependence of the spectrum reveals that the contribution of the topological regions to the DOS overwhelms the trivial contribution in this model. The topological part has a much wider $p_{x}$ region close to its extremal point; therefore, the curvature of the dispersion curve is much smaller than that of the trivial region, where the curvature changes fast. From the conventional theory of magnetic oscillations [30], the 


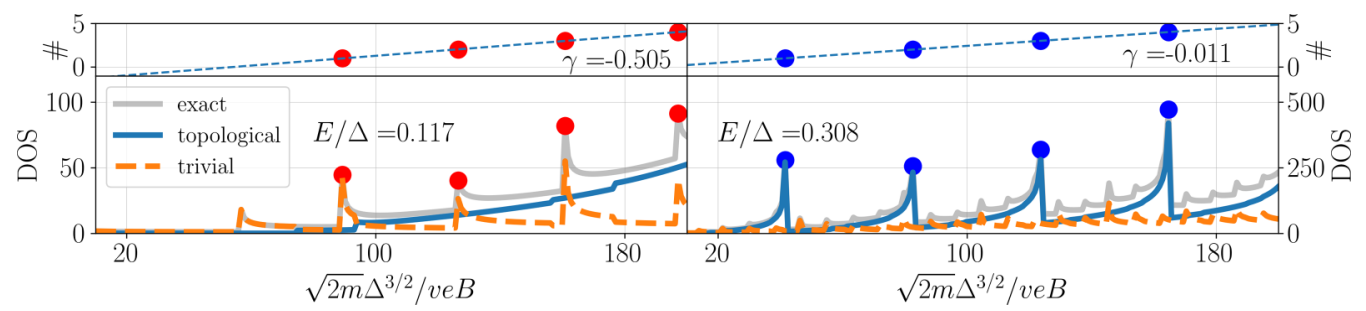

FIG. 4. Magnetic oscillations obtained by the numerical solution of Eq. (2) (exact DOS), as well as from the Onsager approach (topological and trivial DOS) as the function of the inverse magnetic field parallel to the $x$ axis with numerically extracted $\gamma$. For small energies the oscillations of this magnetic interval are dominated by trivial oscillations (left panel), while either increasing energy (right panel) or decreasing the magnetic field the oscillations will be dominated by oscillations of topological origin. Note that, by working at a fixed particle number as opposed to a fixed energy, $\gamma$ is expected [40] to be shifted by an additional $\pm 1 / 8$.

DOS contribution of a given region is inversely proportional to the curvature close to an extremal point; thus the topological contribution dominates the low energy part of the DOS. Upon increasing the magnetic field strength the topological oscillations shift up in energy and give way to trivial ones.

As we demonstrate in Fig. 4, topological and trivial contributions to the DOS can be disentangled. By following the evolution of the peaks in the DOS at fixed energy as a function of the inverse magnetic field, we find periodic structures, as expected. These allow for the extrapolation back to the $n=0$ magnetic quantum number, revealing the underlying topological structure. Indeed, in accord with our previous
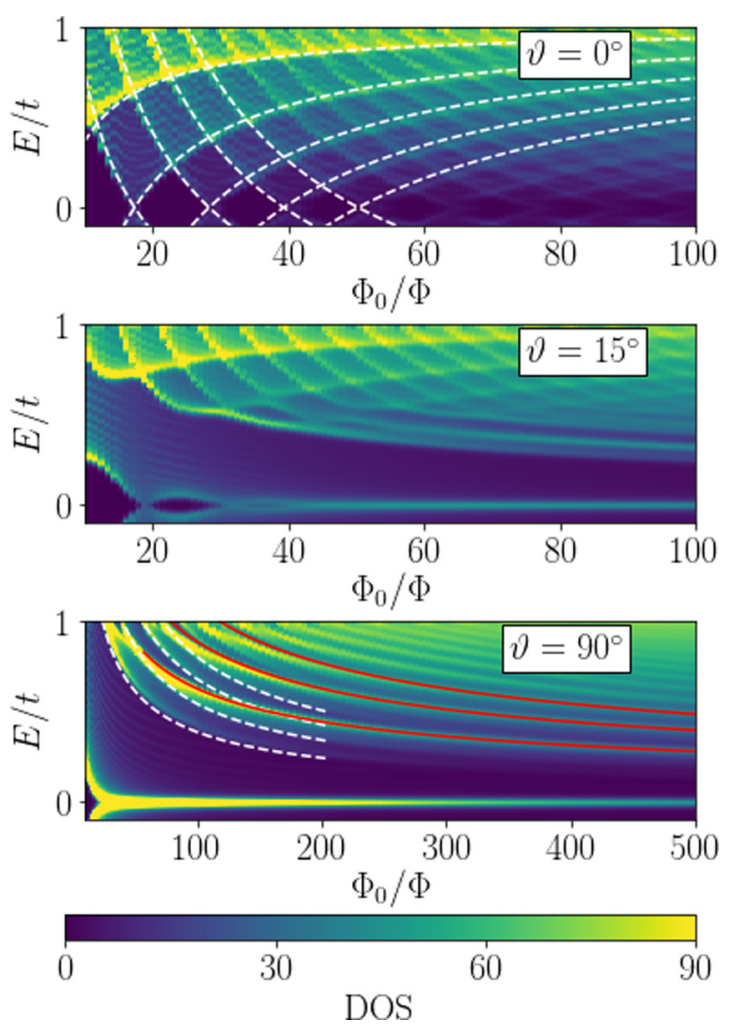

FIG. 5. Density of states for various magnetic field orientations. Dashed white lines for $\vartheta=0$ stem from the solution of the Peierls substituted Eq. (2) with $\vartheta=0$, while the dashed white and solid red lines arise from Eq. (6) using $\Phi / \Phi_{0} \sim B$ and $\Phi_{0}$ is the flux quantum. Already for a small $\vartheta=15^{\circ}$, the features from the topological oscillations are visible. arguments, we find both topological (with Berry phase $\pm \pi$, $\gamma=0$ ) and trivial (with Berry phase zero, $\gamma= \pm 1 / 2$ ) magnetic oscillations, superimposed on top of each other.

In order to underline the robustness of our arguments we performed tight binding calculations based on the lattice Hamiltonian $H_{\mathrm{TB}}=\left(\delta-2 t \sum_{i} \cos \left(k_{i}\right)\right) \sigma_{x}-2 t \sin \left(k_{z}\right) \sigma_{z}$, hosting a nodal loop as in Eq. (2). In all calculations we set $\delta=5 t$ and a finite cubic lattice with 150 unit cells in each direction was taken. Calculations were performed by employing the kernel polynomial method [43,44], including the magnetic field through a Peierls flux $\Phi$. The obtained DOS for various magnetic field orientations is depicted in Fig. 5. These obtained oscillation patterns are in agreement with our previous arguments [45].

\section{CONCLUSIONS}

In summary we have shown that the quantum oscillations in nodal loop semimetals exhibit a peculiar behavior. While the oscillations for magnetic field perpendicular to the plane of the torus are trivial (i.e., with Berry phase zero), a transition occurs upon tilting the magnetic field, where the quantum oscillations consist of coexisting topological and nontopological oscillations with Berry phase $\pi$ and zero, respectively. This follows from an analysis of extremal Fermi surfaces, following the conventional theory of magnetic oscillations, supplemented with a topological inspection of the resulting cross sections. We emphasize that one can easily mix different (i.e., topological and trivial) magnetic peak sequences from the same band by looking at magnetic oscillation patterns in, e.g., the DOS or magnetoresistance and identify unphysical Berry phase contributions. Therefore, it is of great importance to develop an analytical understanding, such as our work, for analyzing existing and future experiments on nodal line and other semimetals.

Note added. Recently, we became aware of related works $[46,47]$. Overlapping results are in agreement.

\section{ACKNOWLEDGMENTS}

This research is supported by the National Research, Development and Innovation Office-NKFIH within the Quantum Technology National Excellence Program (Project No. 20171.2.1-NKP-2017-00001), No. K105149, No. K108676, No. SNN118028, No. K119442, No. K115608, No. K115575, and No. FK124723 and by Romanian UEFISCDI, Project No. 
PN-III-P4-ID-PCE-2016-0032. A.C. acknowledges financial support through the MINECO/AEI/FEDER, UE Grant No. FIS2015-73454-JIN, and the Comunidad de Madrid MAD2D-
CM Program (No. S2013/MIT-3007) L.O. acknowledges the Bolyai program of the Hungarian Academy of Sciences. Calculations were performed on the NIIF cluster.
[1] A. A. Burkov, M. D. Hook, and L. Balents, Phys. Rev. B 84, 235126 (2011).

[2] C. Fang, Y. Chen, H.-Y. Kee, and L. Fu, Phys. Rev. B 92, 081201(R) (2015).

[3] J. K. Asbóth, L. Oroszlány, and A. Pályi, A Short Course on Topological Insulators (Springer International Publishing, Berlin, 2016).

[4] C. Fang, H. Weng, X. Dai, and Z. Fang, Chin. Phys. B 25, 117106 (2016).

[5] N. P. Armitage, E. J. Mele, and A. Vishwanath, Rev. Mod. Phys. 90, 015001 (2018).

[6] J.-W. Rhim and Y. B. Kim, Phys. Rev. B 92, 045126 (2015).

[7] J. Liu and L. Balents, Phys. Rev. B 95, 075426 (2017).

[8] W. B. Rui, Y. X. Zhao, and A. P. Schnyder, Phys. Rev. B 97, 161113(R) (2018).

[9] J. González and R. A. Molina, Phys. Rev. B 96, 045437 (2017).

[10] K. Mullen, B. Uchoa, and D. T. Glatzhofer, Phys. Rev. Lett. 115, 026403 (2015).

[11] H. Huang, J. Liu, D. Vanderbilt, and W. Duan, Phys. Rev. B 93, 201114(R) (2016).

[12] Q. Xu, R. Yu, Z. Fang, X. Dai, and H. Weng, Phys. Rev. B 95, 045136 (2017).

[13] Y. Quan, Z. P. Yin, and W. E. Pickett, Phys. Rev. Lett. 118, 176402 (2017).

[14] M. Hirayama, R. Okugawa, T. Miyake, and S. Murakami, Nat. Commun. 8, 14022 (2017).

[15] Y. Du, F. Tang, D. Wang, L. Sheng, E.-j. Kan, C.-G. Duan, S. Y. Savrasov, and X. Wan, npj Quantum Mater. 2, 3 (2017).

[16] L.-K. Lim and R. Moessner, Phys. Rev. Lett. 118, 016401 (2017).

[17] Y. Kim, B. J. Wieder, C. L. Kane, and A. M. Rappe, Phys. Rev. Lett. 115, 036806 (2015).

[18] R. Yu, H. Weng, Z. Fang, X. Dai, and X. Hu, Phys. Rev. Lett. 115, 036807 (2015).

[19] G. Bian, T.-R. Chang, R. Sankar, S.-Y. Xu, H. Zheng, T. Neupert, C.-K. Chiu, S.-M. Huang, G. Chang, I. Belopolski et al., Nat. Commun. 7, 10556 (2016).

[20] M. Neupane, I. Belopolski, M. M. Hosen, D. S. Sanchez, R. Sankar, M. Szlawska, S.-Y. Xu, K. Dimitri, N. Dhakal, P. Maldonado et al., Phys. Rev. B 93, 201104 (2016).

[21] S. A. Ekahana, S.-C. Wu, J. Jiang, K. Okawa, D. Prabhakaran, C.-C. Hwang, S.-K. Mo, T. Sasagawa, C. Felser, B. Yan et al., New J. Phys. 19, 065007 (2017).

[22] X.-B. Wang, X.-M. Ma, E. Emmanouilidou, B. Shen, C.-H. Hsu, C.-S. Zhou, Y. Zuo, R.-R. Song, S.-Y. Xu, G. Wang et al., Phys. Rev. B 96, 161112 (2017).

[23] J. Hu, Z. Tang, J. Liu, X. Liu, Y. Zhu, D. Graf, K. Myhro, S. Tran, C. N. Lau, J. Wei et al., Phys. Rev. Lett. 117, 016602 (2016).

[24] E. Emmanouilidou, B. Shen, X. Deng, T.-R. Chang, A. Shi, G. Kotliar, S.-Y. Xu, and N. Ni, Phys. Rev. B 95, 245113 (2017).

[25] J. Zhang, M. Gao, J. Zhang, X. Wang, X. Zhang, M. Zhang, W. Niu, R. Zhang, and Y. Xu, Front. Phys. 13, 137201 (2017).
[26] S. Pezzini, M. R. van Delft, L. M. Schoop, B. V. Lotsch, A Carrington, M. I. Katsnelson, N. E. Hussey, and S. Wiedmann, Nat. Phys. 14, 178 (2018).

[27] M. Matusiak, J. R. Cooper, and D. Kaczorowski, Nat. Commun. 8, 15219 (2017).

[28] J. Hu, Z. Tang, J. Liu, Y. Zhu, J. Wei, and Z. Mao, Phys. Rev. B 96, 045127 (2017).

[29] M. N. Ali, L. M. Schoop, C. Garg, J. M. Lippmann, E. Lara, B. Lotsch, and S. S. P. Parkin, Sci. Adv. 2, e1601742 (2016).

[30] A. A. Abrikosov, Fundamentals of the Theory of Metals (NorthHolland, Amsterdam, 1998).

[31] N. W. Ashcroft and N. D. Mermin, Solid State Physics (Saunders College Publishing, Philadelphia, 1976).

[32] G. P. Mikitik and Y. V. Sharlai, Phys. Rev. Lett. 82, 2147 (1999).

[33] A. Alexandradinata and L. Glazman, Phys. Rev. Lett. 119, 256601 (2017).

[34] K. S. Novoselov, A. K. Geim, S. V. Morozov, D. Jiang, M. I. Katsnelson, I. V. Grigorieva, S. V. Dubonos, and A. A. Firsov, Nature (London) 438, 197 (2005).

[35] Y.Zhang, Y.-W. Tan, H. L. Stormer, and P. Kim, Nature (London) 438, 201 (2005).

[36] G. Montambaux, F. Piéchon, J. Fuchs, and M. O. Goerbig, Eur. Phys. J. B 72, 509 (2009).

[37] G. Montambaux, F. Piéchon, J.-N. Fuchs, and M. O. Goerbig, Phys. Rev. B 80, 153412 (2009).

[38] M. Z. Hasan and C. L. Kane, Rev. Mod. Phys. 82, 3045 (2010).

[39] A. H. Castro Neto, F. Guinea, N. M. R. Peres, K. S. Novoselov, and A. K. Geim, Rev. Mod. Phys. 81, 109 (2009).

[40] H. Murakawa, M. S. Bahramy, M. Tokunaga, Y. Kohama, C. Bell, Y. Kaneko, N. Nagaosa, H. Y. Hwang, and Y. Tokura, Science 342, 1490 (2013).

[41] For all magnetic field orientations, $p_{y}$ only constitutes a trivial shift of the cyclotron orbits but the spectrum will not depend on it.

[42] M. Abramowitz and I. A. Stegun, Handbook of Mathematical Functions with Formulas, Graphs, and Mathematical Tables (Dover, New York, 1964).

[43] A. Weiße, G. Wellein, A. Alvermann, and H. Fehske, Rev. Mod. Phys. 78, 275 (2006).

[44] D. Moldovan, M. Anelkovi, and F. Peeters, pybinding v0.9.4: a Python package for tight-binding calculations, 2017.

[45] Only open source numerical packages such as numpy, scipy, matplotlib, pybinding, and ipyvolume were used to obtain the calculated results. We present our numerical code used, in the form of jupyter notebooks, at https://github.com/oroszl/nodalloopsemimetal

[46] H. Yang, R. Moessner, and L.-K. Lim, Phys. Rev. B 97, 165118 (2018).

[47] C. Li, C. M. Wang, B. Wan, X. Wan, H.-Z. Lu, and X. C. Xie, Phys. Rev. Lett. 120, 146602 (2018). 